\section{A hierarchical dualscale study of bisphenol-A-polycarbonate on a silicon surface: structure, dynamics and impurity diffusion}

\author{
Karen Johnston, ${ }^{* a}$ Risto M. Nieminen ${ }^{b}$ and Kurt Kremer ${ }^{a}$ \\ Received 9th March 2011, Accepted 21st April 2011 \\ DOI: $10.1039 / \mathrm{c} 1 \mathrm{sm} 05410 \mathrm{~d}$
}

\begin{abstract}
A previously developed and studied coarse-grained model is used to investigate the properties of bisphenol-A-polycarbonate (BPA-PC) in contact with the $\mathrm{Si}(001)-(2 \times 1)$ surface. The surface interaction potentials are based on density functional calculations. Both a smooth wall potential and a site-dependent wall potential were used to represent the surface. For both types of surface potential it was found that only the chain ends adsorb and the density profiles and conformations in each case are similar. The site-dependent surface slows the dynamics of the polymer at the interface by an order of magnitude compared to the bulk dynamics for the chain lengths considered. The diffusion of nonadsorbing impurity particles for both surface potentials was investigated and the concentration and dynamics of the impurity particles were analysed.
\end{abstract}

\section{Introduction}

Polymer-ceramic composites are widely used in industry due to their properties such as low density, high strength and, therefore, the interaction of polymers with inorganic materials is of high technological relevance. While generic properties of macromolecules close to surfaces are fairly well understood, this does not at all hold for the interaction/adhesion of specific polymers with specific surfaces. One of the challenges is that the strength of the local adhesion can influence conformations and morphologies over many different length scales.

To understand the chemical bonding contribution to adhesion it is necessary to use a quantum mechanical approach. However, these methods are computationally expensive and cannot be used to model systems with more than a few hundred atoms. To obtain information about the structure of the polymer near the interface it is necessary to use a classical atomistic and eventually a coarse-grained model.

A multiscale approach was previously used to study the adhesion of BPA-PC on the Ni(111) surface..$^{1-3}$ For this system it was found that only the phenylene chain ends can adsorb on the surface. ${ }^{1}$ As a consequence the variation of the chain end allows for changing the melt morphology close to the surface from a binding energy dominated polymer brush-like structure to a packing entropy dominated melt. A DFT study of adsorption of these molecules on aluminium found similar results to the case of nickel ${ }^{4}$ although the adsorption energy on aluminium is lower than for nickel.

${ }^{a}$ Max Planck Institute for Polymer Research, P.O. Box 3148, 55021 Mainz, Germany. E-mail: johnston@mpip-mainz.mpg.de

${ }^{b}$ COMP/Applied Physics, Aalto University, P.O. Box 11100, 00076 AALTO, Finland
While the nickel surface is, therefore, representative of metallic surfaces and important in the context of compact disc production, the adhesion on semi-conducting or insulating surfaces may be qualitatively different. As an example, we consider the highly reactive $\mathrm{Si}(001)-(2 \times 1)$ surface, shown in Fig. 1 . This is a frequently studied surface reconstruction, which is stable from below room temperature up to at least $650 \mathrm{~K}$, and thus serves as a benchmark system where the atomic-scale interactions can be described. BPAPC is extremely important since it is one of the few commodity polymers used in high tech applications. Silicon is a major component of many electronic devices and a potential application of this system is protective coatings. Although the surface of silicon in such devices will covered with impurities, this work is a first step towards the understanding of the adhesion of protective coatings.

In this work the building block approach ${ }^{1}$ is used, where the BPA-PC monomer is treated as four 'beads' or molecules, namely carbonate, isopropylidene and two phenylene beads, as shown in Fig. 2. Density functional theory (DFT) is used to calculate the adsorption energies of benzene, carbonic acid and propane, which correspond to the beads, on the Si surface and

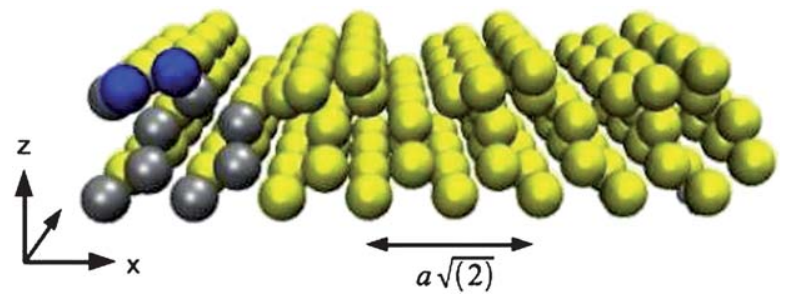

Fig. 1 The $\operatorname{Si}(001)-(2 \times 1)$ surface showing the dimer rows along the $y$ direction. The lattice constant of bulk silicon is $a=5.47 \AA$ and one surface $(2 \times 1)$ cell is highlighted in grey and blue. The two blue atoms in the unit cell comprise one dimer (one adsorption site). 


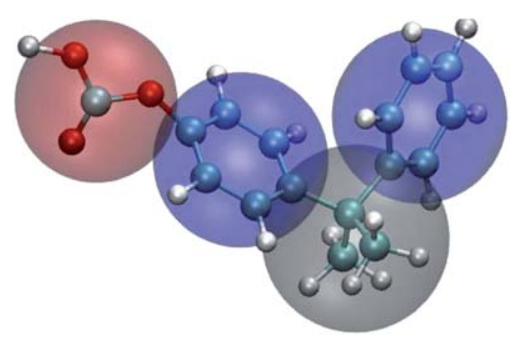

Fig. 2 The monomer of BPA-PC is divided into four beads: two phenylene (blue), one carbonate (red) and one isopropylidene (grey).

these results are used to parameterise the polymer-surface interaction potentials. Only geometries that can be realised within the typical polymer conformations are taken into account.

First-principles results of the molecules on the clean silicon surface $^{5-7}$ showed that benzene adsorbs while the isopropylidene and carbonate groups are repelled from the surface. However, the adsorption of benzene on silicon is qualitatively different to the adsorption on nickel. Benzene in contact with a nickel surface is adsorbed in a flat configuration with the ring remaining undistorted $^{1,8}$ whereas on silicon, benzene is adsorbed on a silicon dimer via a cycloaddition reaction, which distorts the ring structure so that $\theta \approx 120^{\circ}$, as shown in Fig. 3. The interaction of BPA-PC with the clean Si surface is, therefore, expected to be qualitatively different since the benzene bends on approaching the surface so that the isopropylidene and carbonate groups are further away from the surface. This means that the internal phenylene groups may adsorb on the clean $\mathrm{Si}$ surface. Furthermore, there are several adsorption sites on $\mathrm{Ni}$ (111) with comparable adsorption energies, which enables the surface to be described with a smooth (purely $z$-dependent) wall potential, if one is not looking at dynamics. On $\operatorname{Si}(001)-(2 \times 1)$ the phenylene adsorbs only on top of the Si dimers and a smooth wall potential does not describe this surface structure adequately.

This paper uses a coarse-grained classical model to investigate the interaction of the polymer BPA-PC on the highly reactive clean $\mathrm{Si}$ surface. In the next section, we describe the $\mathrm{CG}$ model and the surface-polymer interactions for the smooth surface potential. Later we introduce a site dependence to the surface potential. This model is used to investigate the structure, dynamics and impurity diffusion for these different surface potentials.

\section{Method}

\subsection{Simulation details}

The simulations were performed using the ESPResSo code. ${ }^{9} \mathrm{We}$ simulated a system with 160 chains of 10 monomers ( 43 beads per

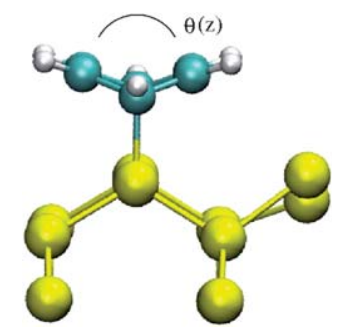

Fig. 3 Benzene adsorbed on the Si surface. chain), which is almost two entanglement lengths for BPA-PC, in a box with sides of length $69.62 \times 69.62 \times 143.23 \AA^{3}$. This corresponds to $9 \times 18$ surface unit cells for both the top and bottom surfaces or a total of 324 adsorption sites (Si dimers). In a melt at $570 \mathrm{~K}$ the typical end-to-end distance for a 10-monomer chain is $<R_{\mathrm{e}}^{2}>^{\frac{1}{2}}=48 \AA$ and the radius of gyration is $<R_{\mathrm{g}}^{2}>^{\frac{1}{2}}=19 \AA$ A. $^{10}$ The extension of a fully stretched chain is about $110 \AA$, keeping the mutual influence of the two surfaces negligible.

The initial polymer configurations were generated using a random walk algorithm and the system was run using a Langevin thermostat with a friction parameter of $0.5 \tau^{-1}$ and a time step of $10^{-3} \tau$. The equilibration time for a bulk melt with 80 chains of length 10 monomers is $6 \times 10^{3} \tau .^{11}$ For the attractive wall simulations we started from a melt confined between repulsive walls that was equilibrated for $10^{5} \tau$.

The DFT calculations were performed with the VASP code. ${ }^{12}$ The PBE generalised gradient functional ${ }^{13-15}$ was used to approximate the exchange and correlation and the core electrons were treated using the projector augmentented wave method (PAW). ${ }^{16,17}$ The DFT equilibrium lattice constant of $5.47 \AA$ was used for both the DFT calculations and for the coarse-grained model. The surface was represented by 5 atomic layers of silicon, with the bottom surface passivated with hydrogen atoms, in a periodic cell of depth $\approx 22 \AA$.

The effect of van der Waals (vdW) forces is not included in the DFT calculations. For benzene on silicon, vdW forces increase the adsorption energy by around $0.2 \mathrm{eV} \approx 4 k_{\mathrm{B}} T$ at $570 \mathrm{~K} .{ }^{18} \mathrm{We}$ do not expect the isopropylidene and carbonate groups to experience stronger vdW forces than the phenylene group since they are less polarisable. In atomistic molecular dynamics simulations of bulk BPA-PC (50 5-monomer chains, using the model in Hess et al. ${ }^{19}$ ) the Lennard-Jones interactions per bead are $\approx 18 \mathrm{~kJ} \mathrm{~mol}^{-1} \approx 3.8 k_{\mathrm{B}} T$, which is of the same magnitude as the vdW contribution to the adsorption energy. Since the coarsegrained model of bulk BPA-PC neglects vdW interactions, the $\mathrm{vdW}$ interactions with the surface can also be neglected without changing the energy balance between the surface and bulk interactions.

\subsection{Bulk interactions}

In this subsection we describe the coarse-grained model for bulk BPA-PC that was developed by Abrams et al. ${ }^{11}$ Bonded interactions are represented by harmonic potentials of the form

$$
V(r)=\frac{1}{2} k_{r, i j}\left(r-r_{i j}\right)^{2}
$$

where $r_{\mathrm{CP}}=3.56 \AA$ for the carbonate-phenylene bond, $r_{\mathrm{IP}}=2.93$ $\AA$ for the isopropylidene-phenylene bond and $k_{r, i j}=625 k_{\mathrm{B}} T$ $\AA^{-2}$. For the angle potentials PĈP and PÎP we used the tabulated potentials described in ref. 11. The angle IPC was described by a stiff harmonic potential of the form

$$
V(\theta)=\frac{1}{2} k_{\theta}\left(\theta-\theta_{0, \mathrm{IPC}}\right)^{2}
$$

where $k_{\theta}=0.1 k_{\mathrm{B}} T / \mathrm{deg}^{2}$ and $\theta_{0, \mathrm{IPC}}=\pi$. Torsion potentials were neglected since the torsion barriers are less than $k_{\mathrm{B}} T$. 
Excluded volume interactions are described by Weeks-Chandler-Anderson (WCA) potentials of the form

$$
V_{\mathrm{LJ}}(r)=4 \varepsilon\left\{\left(\frac{\sigma}{r}\right)^{12}-\left(\frac{\sigma}{r}\right)^{6}+c_{\text {shift }}\right\}, r<r_{\text {cut }}
$$

where $\varepsilon=1, c_{\text {shift }}=0.25$ and $r_{\text {cut }}=2^{\frac{1}{6}} \sigma$. The bead diameters are $\sigma_{\mathrm{C}}=3.49 \AA, \sigma_{\mathrm{P}}=5.19 \AA$ and $\sigma_{\mathrm{I}}=4.67 \AA$ for the carbonate, phenylene and isopropylidene groups, respectively, and arithmetic mixing rules are used. WCA interactions between nearest neighbours and P-P next-nearest neighbours for internal beads are excluded.

This CG model has been used to study the structural and dynamical properties of bulk BPA-PC. ${ }^{10}$ In the study of BPA-PC on nickel ${ }^{1}$ and in the present case of BPA-PC on silicon, the parameters of this $\mathrm{CG}$ model remain unchanged. To describe the interaction with the surface we develop additional surface potentials that are based on the results of DFT calculations. The following subsections describe the development of the surface interaction for two types of surface potential, namely, a smooth wall potential and a site-dependent potential.

\subsection{Smooth surface interactions}

For the smooth surface the potentials depend only on the distance from the surface, $z$. The site-dependent surface potential, which varies also in the $x y$-plane, will be introduced in the following subsection. From density functional calculations ${ }^{5-7}$ we know that benzene is chemically bonded to the clean $\mathrm{Si}(001)-(2 \times$ 1) surface with an adsorption energy of $E_{\mathrm{ads}}=1.04 \mathrm{eV}$ (equivalent to $21.2 \mathrm{k}_{\mathrm{B}} \mathrm{T}$ at $570 \mathrm{~K}$ ). In equilibrium the benzene is at a distance of $z_{\text {eq }}=2.2 \AA$ from the surface and the interaction goes to zero at $z_{\max }=3.1 \AA$. This interaction is represented by a 9-3 Lennard-Jones/cosine non-bonded interaction of the form

$$
U_{\mathrm{PW}}(z)=\left\{\begin{array}{l}
\frac{E_{\mathrm{ads}}}{s}\left[a_{P}\left(\frac{\sigma_{\mathrm{PW}}}{z-z_{\mathrm{eq}}+z_{0}}\right)^{9}\right. \\
\left.-b_{P}\left(\frac{\sigma_{\mathrm{PW}}}{z-z_{\mathrm{eq}}+z_{0}}\right)^{3}+c_{\mathrm{P}}\right] \quad z \leq z_{\mathrm{eq}} \\
\frac{E_{\mathrm{ads}}}{2}\left[\cos \left[\pi \frac{z+z_{\mathrm{max}}-2 z_{\mathrm{eq}}}{z_{\mathrm{max}}-z_{\mathrm{eq}}}\right]-1\right] \quad z_{\mathrm{eq}}<z<z_{\max } \\
0 \quad z \geq z_{\max }
\end{array}\right.
$$

where

$$
\begin{gathered}
s=\frac{2 b_{\mathrm{P}}}{\left(3 \sqrt{\left(3 a_{\mathrm{P}} / b_{\mathrm{P}}\right)}\right)}, \\
z_{0}=\sigma_{\mathrm{PW}}\left(\frac{3 a_{\mathrm{P}}}{b_{\mathrm{P}}}\right)^{\frac{1}{6}}, \\
a_{\mathrm{P}}=b_{\mathrm{P}}\left(\frac{z_{\mathrm{min}}-z_{\mathrm{eq}}}{\sigma_{\mathrm{PW}}\left(1-3^{\frac{1}{6}}\right)}\right)^{6},
\end{gathered}
$$

$b_{\mathrm{P}}=100, c_{\mathrm{P}}=0, z_{\text {min }}=1.5 \AA$ and $\sigma_{\mathrm{PW}}=2.0 \AA$. The potential is shown in Fig. 4.
An important question is whether benzene adsorption is really representative of the phenylene adsorption i.e. how good is the "building block" approximation? To assess if the electronic structure of adsorbed benzene is significantly different to phenylene, DFT calculations were performed where a hydrogen atom of the benzene is replaced by a carbonyl or isopropylidene group and the adsorption energies are compared. It was found that phenylene with a carbonate group attached $\left(\mathrm{HCO}_{3}-\mathrm{C}_{6} \mathrm{H}_{5}\right)$, denoted $\mathrm{PC}$, has the same adsorption energy as benzene i.e. 1.04 eV. A phenylene with isopropylidene attached $\left(\mathrm{C}_{3} \mathrm{H}_{7}-\mathrm{C}_{6} \mathrm{H}_{5}\right)$, denoted PI, has a somewhat lower adsorption energy of $0.89 \mathrm{eV}$ but this difference is due to the fact that this larger molecule is repelled more strongly from the surface, simultaneously distorting the phenylene ring from its optimum structure.

The other components of the polymer chain, namely the isopropylidene and the carbonate groups are represented by repulsive potentials chosen to match the DFT results. Since the carbonic acid and propane molecules dissociate on contact with the surface it is necessary to either (a) constrain the molecule or (b) consider a larger segment of the monomer. As mentioned already method (b) includes an additional energy contribution from the distortion of the phenylene bead so method (a) gives more reliable data. Although the carbonate and phenylene groups are more stable within the chain than as isolated molecules, we cannot rule out the possibility that in some conformations they could dissociate on the surface. However, we believe that this scenario does not play a dominant role and this work excludes the possibility of dissociation. Starting from the minimum energy structure for PC/PI on $\mathrm{Si}$, the phenylene group was replaced by a hydrogen atom and the interaction between the molecule (C/I) and the surface was calculated. The molecule was gradually moved along $\mathrm{z}$, keeping all other molecular coordinates fixed. The data are shown in Fig. 4. For the repulsive potentials we fitted $93-\mathrm{LJ}$ potentials of the form

$U_{\mathrm{JW}}(z)=4 \varepsilon\left[a_{\mathrm{J}}\left(\frac{\sigma_{\mathrm{J}}}{z-z_{\text {cutJ }}+z_{0}}\right)^{9}-b_{\mathrm{J}}\left(\frac{\sigma_{\mathrm{J}}}{z-z_{\text {cut }}+z_{0}}\right)^{3}+c_{\mathrm{J}}\right] z<z_{\text {cut }}$

where $\mathrm{J}$ is either the $\mathrm{C}$ or $\mathrm{I}$ bead, $\varepsilon=1, a_{\mathrm{C}}=1106, a_{\mathrm{I}}=694.4$, $b_{\mathrm{C}}=39.85, b_{\mathrm{I}}=18.22$,

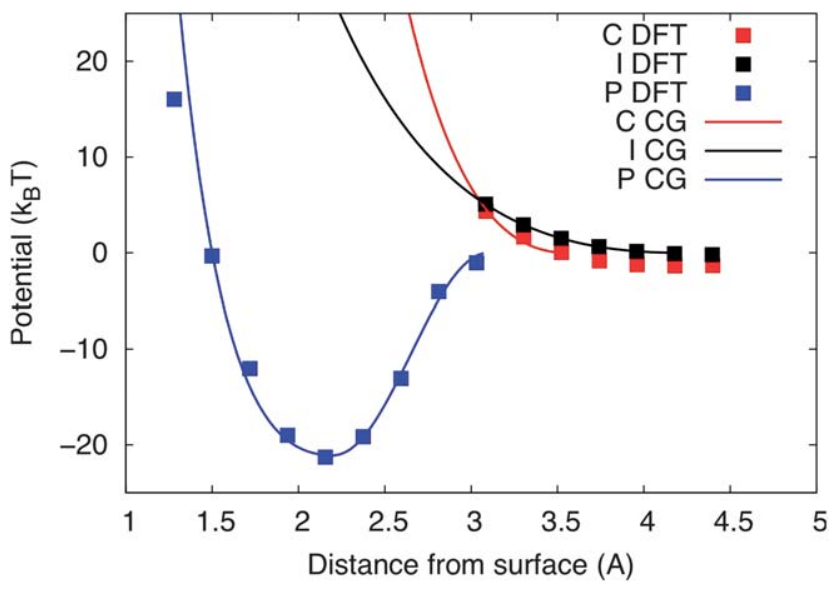

Fig. 4 Surface potentials for P, C and I beads and the corresponding DFT results. 


$$
\begin{array}{r}
c_{\mathrm{J}}=\frac{2}{3 \sqrt{3}} \sqrt{\frac{b_{\mathrm{J}}^{3}}{a_{\mathrm{J}}}}, \\
z_{\text {cutC }}=3.55 \AA, z_{\text {cutI }}=4.2 \AA \text { and } \\
z_{0 \mathrm{~J}}=\sigma_{\mathrm{J}}\left(\frac{3 a_{\mathrm{J}}}{b_{\mathrm{J}}}\right)^{\frac{1}{6}} .
\end{array}
$$

The potentials for the three beads are shown in Fig. 4, alongside the DFT results.

In addition to the adsorption energies, we know that the phenylene ring bends on adsorption, as shown in Fig. 3. Here we account for this "butterfly" adsorption structure by allowing the IPC angle potential to vary with the distance from the surface

$$
U_{\mathrm{IPC}}(\theta, z)=\frac{1}{2} k_{\theta}\left(\theta-\theta_{0, \mathrm{IPC}}(z)\right)^{2}
$$

where the equilibrium angle $\theta_{0}$, IPC depends on the distance $z$ from the surface

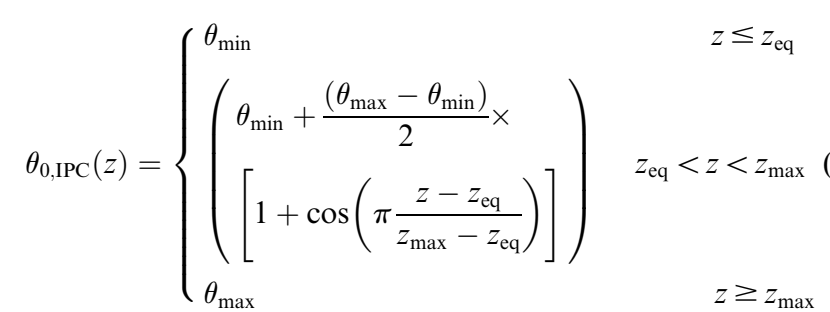

and where $\theta_{\text {min }}=120^{\circ}, \theta_{\text {max }}=180^{\circ}, z_{\text {max }}=3.1 \AA z_{\text {eq }}=2.2 \AA$ as before. The force constant, $k_{\theta}$, is assumed to be the same as for the IPC angle in the bulk melt. This potential is equal to the intrachain IPC bending potential of bulk BPA-PC when $z \geq z_{\max }$. A similar potential is applied to the chain ends where the angular potential is between the surface normal and the $\mathrm{P}-\mathrm{C}$ bond. In this case it is necessary to smoothly switch on the potential as the chain end approaches the surface. The potential is given by

$$
U_{\mathrm{PCW}}(\theta, z)=\frac{1}{2} f(z) k_{\theta}\left(\theta-\theta_{0, \mathrm{PCW}}(z)\right)^{2}
$$

where the switching function is

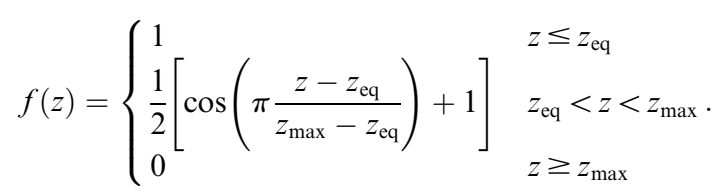

and $\theta_{0, \operatorname{PCW}}(z)$ has the same form as $\theta_{0, \operatorname{IPC}}(z)$ but with $\theta_{\min }=60^{\circ}$ and $\theta_{\max }=90^{\circ}$. The equilibrium angles, switching function and potentials are shown in Fig. 5. So far, the energetics of the optimal adsorption site is described. For the flat surface we take this as a homogeneous $z$-dependent surface potential.

\subsection{Site-dependent surface interactions}

In the $\operatorname{Si}(001)-(2 \times 1)$ surface there are specific adsorption sites, namely the Si dimers, which could significantly influence the structure and dynamics of the polymer at the surface. First, neighbouring phenylene beads will only be able to adsorb if the energy due to the angular distortion (i.e. for the PÎP and PCP angles) is not higher than the adsorption energy. Second, the site dependence will affect the diffusion of $\mathrm{P}$ beads along the surface. Here, we only introduce the site dependence for the phenylene beads and keep the smooth repulsive potentials for the carbonate and isopropylidene beads. This is not an unrealistic approximation since only the phenylene beads (benzene) adsorb and, therefore, show a strong site dependence.

The surface unit cell has a lattice constant of $a_{0}=5.47 \sqrt{2} \AA$ and cell vectors of $(1,0)$ and $(0,0.5)$. The dimer sites are on the corners of the cell at $(0,0)$, as shown in Fig. 6(a). With just the attractive LJ-cosine potential $U_{\mathrm{PW}}(r)$ on the dimer sites, and for small distances from the surface the potential minimum occurs between the nearest neighbour dimers at $(0,0.5)$, shown in Fig. 6 (c). Since it is known that benzene adsorbs only on top of the dimer sites it is necessary to compensate for these unphysical potential wells. Therefore, repulsive cosine potentials of the form

$$
U_{\text {rep }}(r)=A\left[1+\cos \left(\frac{\pi r}{r_{\text {cut }}}\right)\right]
$$

were added to three additional sites $(0,0.5,0),(0.25,0,0)$ and $(0.75,0,0)$ in the surface unit cell, as shown in Fig. 6(a). Each repulsive potential was constructed to go to zero at a radius of $r_{\text {cut }}=2.5 \AA$. The effect of adding the repulsive potentials is shown in Fig. 6(c) and (d). The values of $A$ are $40 k_{\mathrm{B}} T$ on site $(0,0.5)$ and $20 k_{\mathrm{B}} T$ on sites $(0.25,0,0)$ and $(0.75,0,0)$. The resulting potential energy landscape at $\mathrm{z}=2.2 \AA$ from the surface is shown in Fig. 6(b). At this distance from the surface the barrier between the sites is the same as the well depth of $\approx 20 \mathrm{kT}$. For distances further from the surface, i.e. $z_{\max } \geq 2.2 \AA$, but still within the range of the attractive potential $z_{\max } \leq 3.1 \AA$, the barrier between sites is smaller.

\section{Results}

\subsection{Adsorption}

Once the attractive potentials are switched on adsorption happens rather quickly, as shown in Fig. 7(a). A bead is considered to be adsorbed if it is less than $3 \AA$ from the surface. Once a bead adsorbs on a surface it rarely desorbs and, therefore, the system never truly equilibrates. This is especially important for the site-dependent surface potential since adsorption sites will be blocked. Nevertheless, after $400 \times 10^{3} \tau$ the adsorption rate is very slow and the system reaches a saturation coverage. This time corresponds to a mean square displacement of all beads of $10^{4}-10^{5} \AA^{2}$, which means that the particles in a melt would have travelled a distance of the same order of magnitude as the box size. ${ }^{10}$ The structure and dynamics of the melt are analysed after this time. For the site-dependent potential an adsorbed bead will remain stuck for most of the time on an adsorption site whereas for the smooth surface the adsorbed beads are free to slide around on the surface. This implies that for the site-dependent cases the final structure may depend somewhat on the initial configuration i.e. when a bead adsorbs it is likely to remain in that position remainder of the simulation. For this reason two independent site-dependent calculations with different starting configurations are considered.

For the smooth potential the rate of adsorption is greater than for the site-dependent potential since the beads see the attractive surface at all points along the $x y$ plane. From DFT calculations 

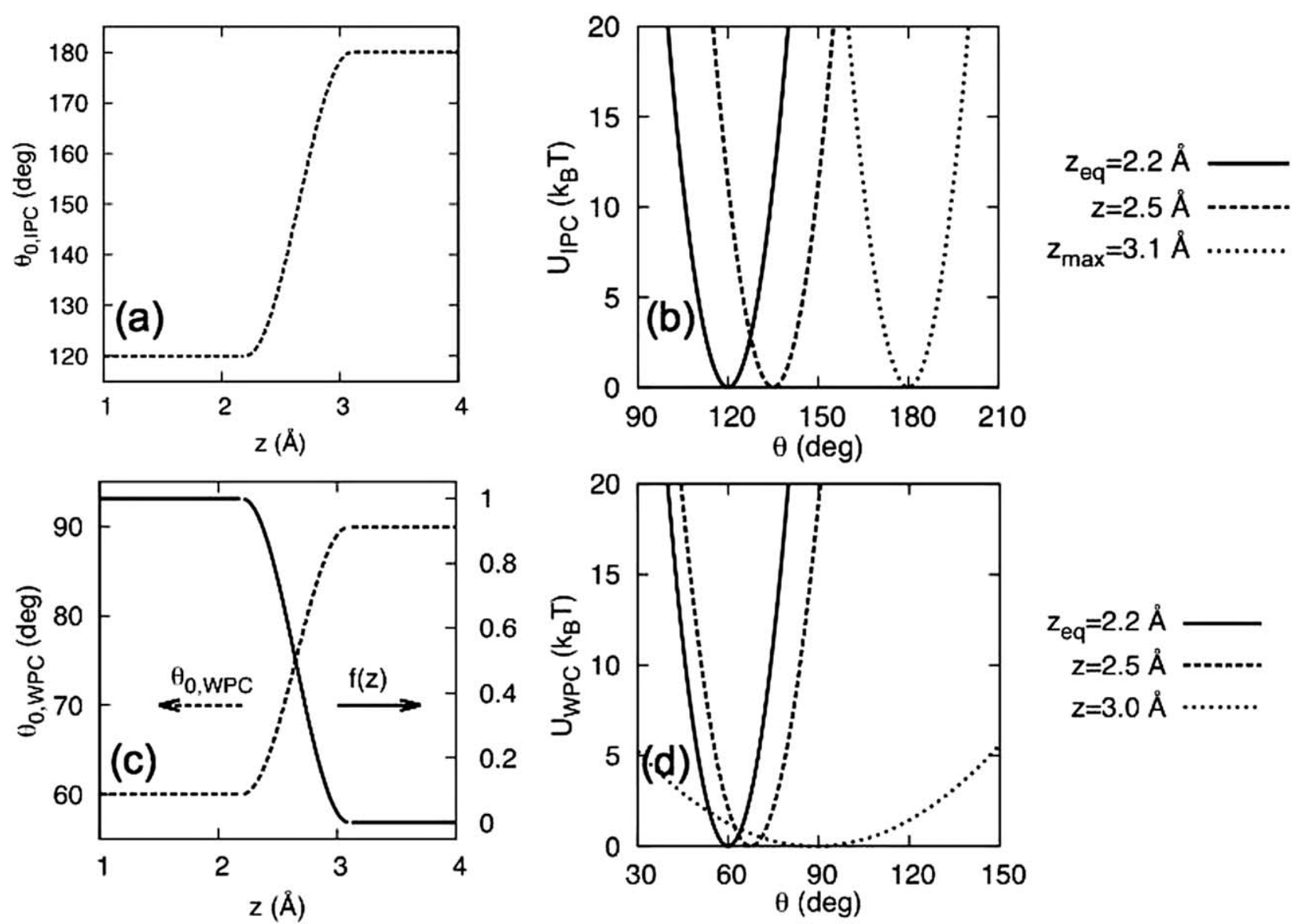

Fig. 5 Angle-distance harmonic potentials for IPC and WPC angles. (a) For the IPC angle the value of $\theta_{0}$, IPC changes smoothly from $180^{\circ}$ to $120^{\circ}$ as the $\mathrm{P}$ bead approaches the surface. (b) Although $\theta_{0}$, IPC changes the force constant is independent of distance. (c) For the WPC angle $\theta_{0}$, PCW changes and the force constant is modified by $f(z)$. (d) The WPC angle potential becomes flatter as $z \rightarrow z_{\max }$ and is zero for $z \geq z_{\max }$.

of benzene on the silicon surface it is known that a benzene coverage of 0.5 monolayers (ML), which corresponds to one benzene molecule on every other adsorption site, is stable but a 1.0 ML coverage is unstable (negative adsorption energy), due to repulsion between neighbouring benzene molecules. For the smooth surface the number of adsorbed beads exceeds $0.5 \mathrm{ML}$ but the systems with site dependence saturate at a coverage approaching $0.5 \mathrm{ML}$.

This coverage of $0.5 \mathrm{ML}$ for the site-dependent potential is at first surprising since it implies that there are no defects in the coverage (i.e. exactly every other site is occupied). However, this is not the case and the coverage is not perfectly ordered, as shown in Fig. 7(b), with some neighbouring sites being occupied despite this proximity being unstable in density functional calculations. The occupancy of neighbouring sites is due to the fact that the coarse-grained model does not describe all the details of adsorption, such as directionality of the bonding, surface strain effects, etc. Nevertheless, the adsorption along the dimer rows is clearly visible in Fig. 7(b). This is clearly different to the case of adsorption on $\mathrm{Ni}(111)$, which is shown in Fig. 10 of Andrienko et al. ${ }^{20}$ In systems with longer polymer chains fewer chain ends are available and, therefore, the coverage might be expected to be lower than in the current case. However, in experiment the polydispersity can be high and it is known from simulation that short chains aggregate on the surface. ${ }^{20}$ We would, therefore, expect that the current coverage is relevant also in experiment.

\subsection{Density profiles}

The densities are averaged over a time period of $50 \times 10^{3} \tau$ and are shown in Fig. 8. The densities are normalised relative to the total bulk density. There are several peaks and troughs near the surface and the density does not become constant and bulk-like until around $20 \AA$. This value is similar to the value found for other polymer melts in contact with surfaces, for example, polystyrene on graphite ${ }^{21}$ and poly(ethylene oxide) on $\mathrm{TiO}_{2}{ }^{22}$ The density profile is almost identical to that of BPA-PC on nickel,${ }^{20}$ which is not surprising since the surface interaction is dominated by end effects. The total density as a function of $z$ for the different wall potentials are shown in the inset of Fig. 8. The density at the surface has a high peak corresponding to the adsorbed beads and is higher for the smooth potential than for the site-dependent potential as seen before in Fig. 7(a).

The density profile for the different components of BPA-PC is shown in Fig. 8. The first peak in the total density is clearly due to the $\mathrm{P}$ beads that are adsorbed on the surface. The second peak is mainly due to the $\mathrm{C}$ beads and the third is due mostly to $\mathrm{P}$ beads. The peak at the surface is composed of $\mathrm{P}$ chain ends only, i.e. no internal $\mathrm{P}$ beads are adsorbed, and the system is depleted in chain 

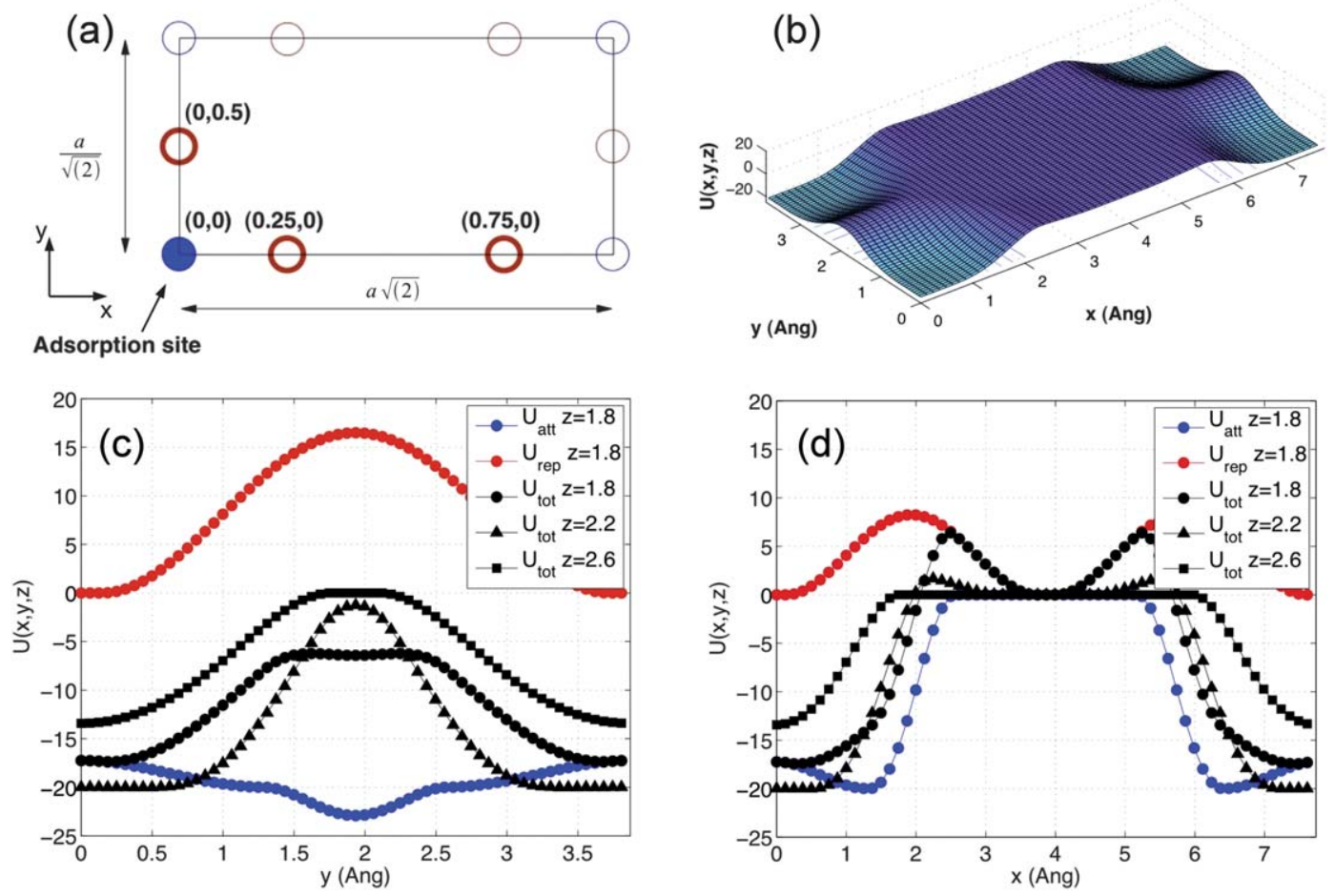

Fig. 6 (a) A surface unit cell showing the centres of the attractive (blue filled circles) and repulsive (red unfilled) sites. (b) The potential energy landscape $\left(k_{\mathrm{B}} T\right)$ in the surface unit cell at $\mathrm{z}=2.2 \AA$ from the surface. The attractive, repulsive and total potentials $\left(k_{\mathrm{B}} T\right)$ along (c) $x=0$ and $(\mathrm{d}) y=0$. Total potentials are shown for several values of $z(\AA)$.

ends until $\approx 50 \AA$ from the surface. Despite the addition of the $z$ dependent angle potential on IPC, the internal beads do not adsorb on the surface, due to the excluded volume of the I and C groups. Adsorption would only be possible for a totally flat approach, which is highly improbable due to chain geometry and packing, as the data show. Qualitatively, the adsorption structure is similar to that on $\mathrm{Ni}(111)$ where only the chain ends bind to the surface. ${ }^{20}$

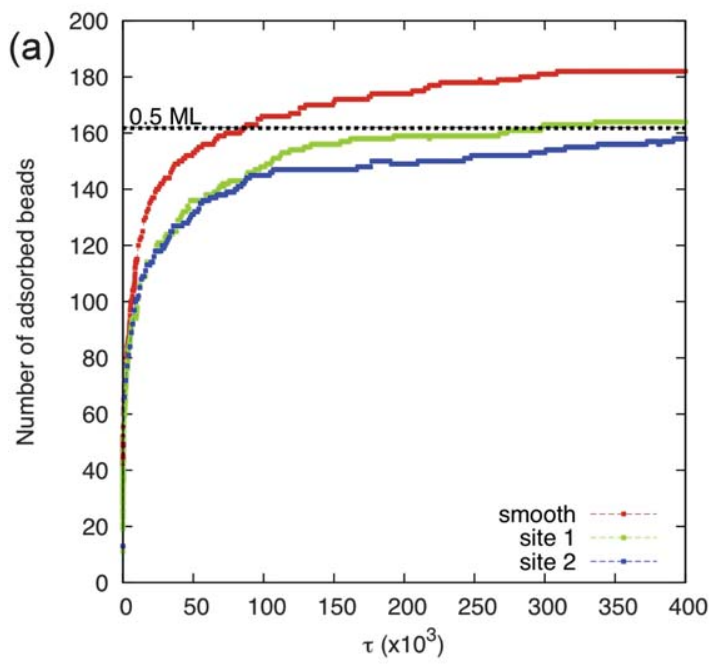

Fig. 9 shows a snapshot of the smooth wall system where we differentiate between chains that are attached on both ends, attached on one end or unattached to any surface. A snapshot of the site-dependent surface looks qualitatively the same except for the adsorbed layer which exhibits ordering along the dimer rows. The properties of the one-end-attached or both-end-attached chains are different to those of the unattached, bulk-like chains. We can calculate the radius of gyration, $\left\langle R_{\mathrm{g}}^{2}\right\rangle$, and the

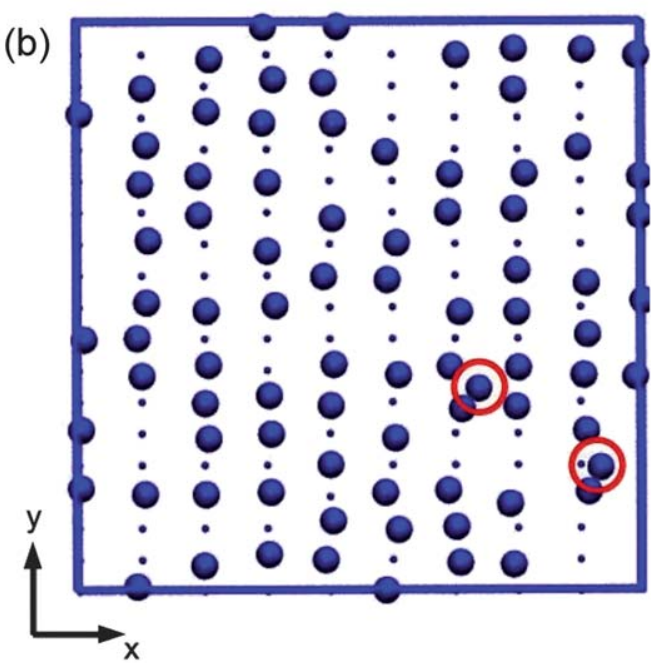

Fig. 7 (a) Number of adsorbed beads $v s$. time $\tau$ for the smooth surface potential and two independent runs with a site-dependent potential. The dashed horizontal line corresponds to a coverage of 0.5 monolayers, which means that one benzene molecule is adsorbed on every other adsorption site. (b) Snapshot showing the adsorption on a surface with a site-dependent potential. The dots represent the adsorption sites and the blue particles are chain ends with $z<3.0 \AA$. The beads highlighted in the red circles are slightly further from the surface than the other beads and are not strongly adsorbed. 


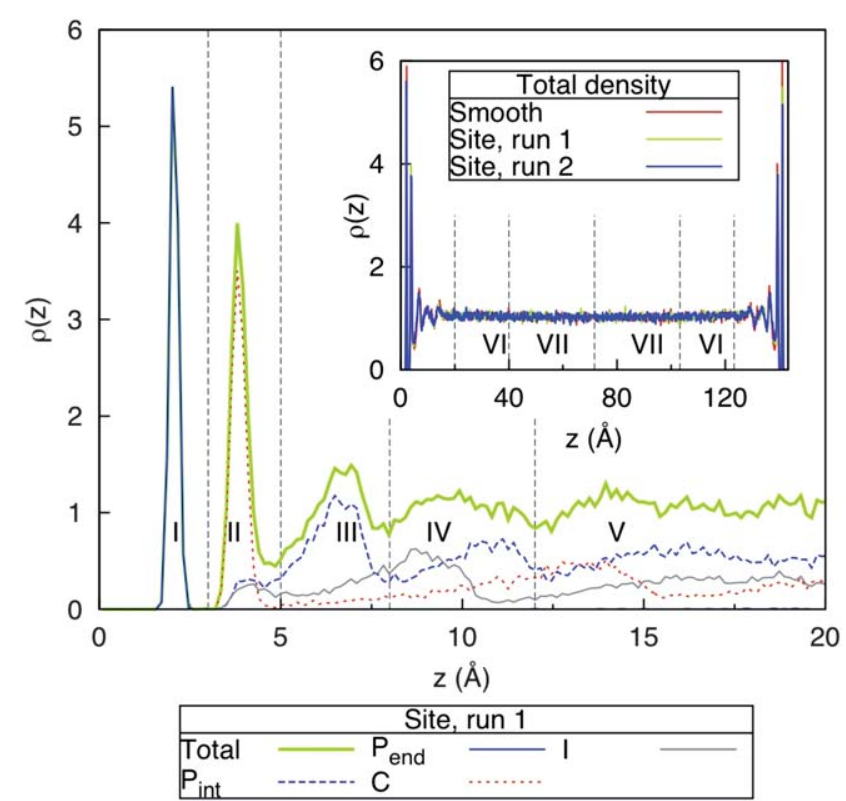

Fig. 8 The densities of the individual components of BPA-PC for one of the site-dependent simulations. The distribution of internal $\mathrm{P}$ beads $\left(\mathrm{P}_{\mathrm{int}}\right)$ and $\mathrm{P}$ chain ends $\left(\mathrm{P}_{\text {end }}\right)$ are shown separately. The inset shows the total density profile of BPA-PC along the $z$ direction for the smooth and sitedependent systems. The densities are normalized so that the total bulk density is equal to 1.0 .

end-to-end distance, $\left\langle R_{\mathrm{e}}^{2}\right\rangle$, for these three types of chain. The values are averaged over 50 configurations that are separated by $1 \times 10^{3} \tau$. For the bulk system the values are $<R_{\mathrm{g}}^{2}>=349 \AA^{2}$ and $\left.<R_{\mathrm{e}}^{2}\right\rangle=2291 \AA^{2}$, which is in excellent agreement with the previous values of $\left\langle R_{\mathrm{g}}^{2}>=348 \AA^{2}\right.$ and $<R_{\mathrm{e}}^{2}>=2270 \AA^{2}{ }^{2}{ }^{10}$ For the surface systems, it is clear that chains with only one end attached

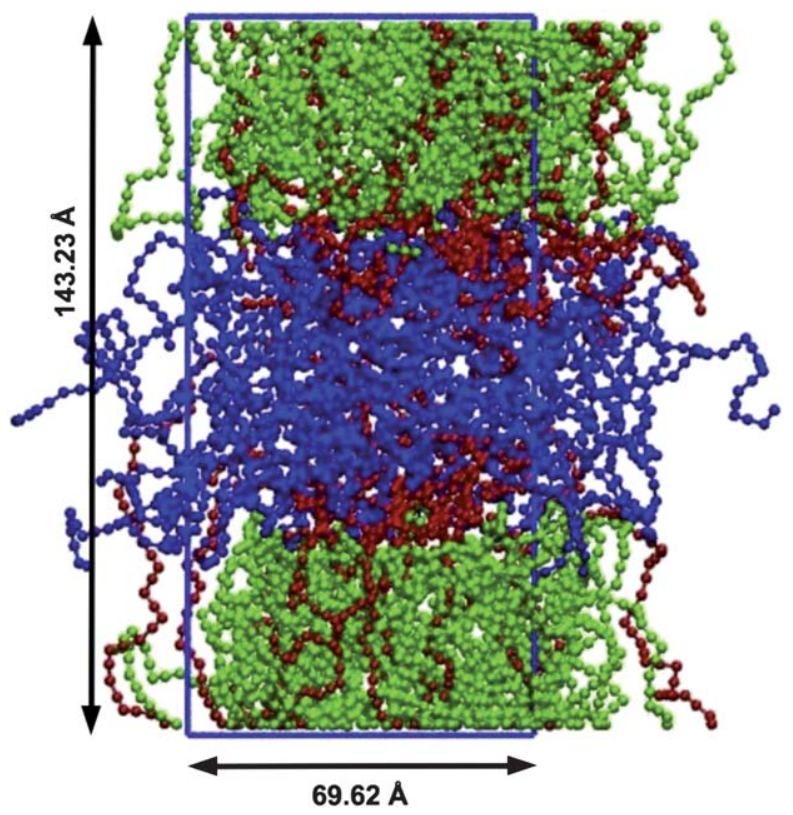

Fig. 9 Snapshot of BPA-PC adsorbed on the smooth wall. Chains with both ends adsorbed are shown in green, chains with one end adsorbed in red and chains that are not adsorbed at all are in blue. The blue box is the simulation cell. have a significantly larger $<R_{\mathrm{e}}>$ than the other chains, which is due to their brush-like conformations (see Fig. 9). Chains with both ends attached have a smaller $\left\langle R_{\mathrm{e}}\right\rangle$ than the other chain types. The unattached chains do not have the same $\left\langle R_{\mathrm{e}}\right\rangle$ or $<R_{\mathrm{g}}>$ as the bulk, which implies that we do not have true bulklike behaviour in the centre of the slab. The $\left\langle R_{\mathrm{e}}\right\rangle$ and $\left\langle R_{\mathrm{g}}\right\rangle$ and their standard deviations are summarised in Table 1.

\subsection{Dynamics}

The relative dynamics of the different systems can be compared by calculating the mean square displacements (msd) of all the beads $g_{1}$, which is given by

$$
\left.g_{1}(t)=<\left|r_{i}(t)-r_{i}\left(t_{0}\right)\right|^{2}\right\rangle
$$

where $i$ is the bead index and $\langle\ldots\rangle$ denotes the ensemble average. The msd for the different systems are shown in Fig. 10(a). For the bulk system (without walls), $g_{1}$ is in good agreement with previous studies of BPA-PC ${ }^{10,19}$ and shows the crossover from $t^{\frac{1}{2}}$ scaling to diffusive behaviour (linear scaling) around $10^{4}-10^{5} \tau$. The dynamics of the smooth wall system is slower than the bulk. This is mainly due to the suppression of dynamics in the $z$-direction as can be seen by comparing the $g_{1}$ of the smooth wall with the $g_{1 x y}$ of the bulk system. The site-dependent systems show an additional slowing down, which reflects the suppression of the dynamics in the $x y$-plane close to the surface. The two different site-dependent systems show the same dynamical behaviour.

To understand in more detail the effect of the interface we analyze the local mobility, where the different layers corresponding to the density peaks are analysed separately. At each time $t$ the msd in $x$ and $y$, denoted $g_{x y}^{(j)}$, are calculated for particles in region $j$. A particle's trajectory only contributes to $g_{x y}^{(j)}$ while it remains in region $j$. The average msd is defined as

$$
g_{1 x y}^{(j)}(\delta t)=\frac{1}{N_{j}} \sum_{i=1}^{N_{j}}\left[\left(x_{i}(t)-x_{i}\left(t_{0}\right)\right)^{2}+\left(y_{i}(t)-y_{i}\left(t_{0}\right)\right)^{2}\right]
$$

Table $1<R_{\mathrm{g}}^{2}>$ and $<R_{\mathrm{e}}^{2}>$ for the different chain types and systems. $\sigma$ is the standard deviation. Units are in $\AA^{2}$

\begin{tabular}{lllll}
\hline & $\left\langle R_{\mathrm{g}}^{2}\right\rangle$ & $\sigma$ & $\left\langle R_{\mathrm{e}}^{2}\right\rangle$ & $\sigma$ \\
\hline Bulk & & & & \\
Total $^{10}$ & 348 & 120 & 2270 & 1160 \\
Total & 349 & 136 & 2291 & 1581 \\
Smooth & & & & \\
Total & 357 & 139 & 2085 & 1812 \\
Unattached & 327 & 131 & 2073 & 1506 \\
One end & 503 & 124 & 4219 & 1652 \\
Both ends & 304 & 92 & 1008 & 886 \\
Site, run 1 & & & & \\
Total & 372 & 150 & 2371 & 1830 \\
Unattached & 323 & 125 & 1994 & 1420 \\
One end & 484 & 135 & 4037 & 1699 \\
Both ends & 341 & 141 & 1576 & 1496 \\
Site, run 2 & & & & \\
Total & 374 & 144 & 2401 & 1739 \\
Unattached & 333 & 133 & 2107 & 1525 \\
One end & 459 & 138 & 3746 & 1742 \\
Both ends & 346 & 128 & 1583 & 1212 \\
\hline & & & & \\
\hline
\end{tabular}



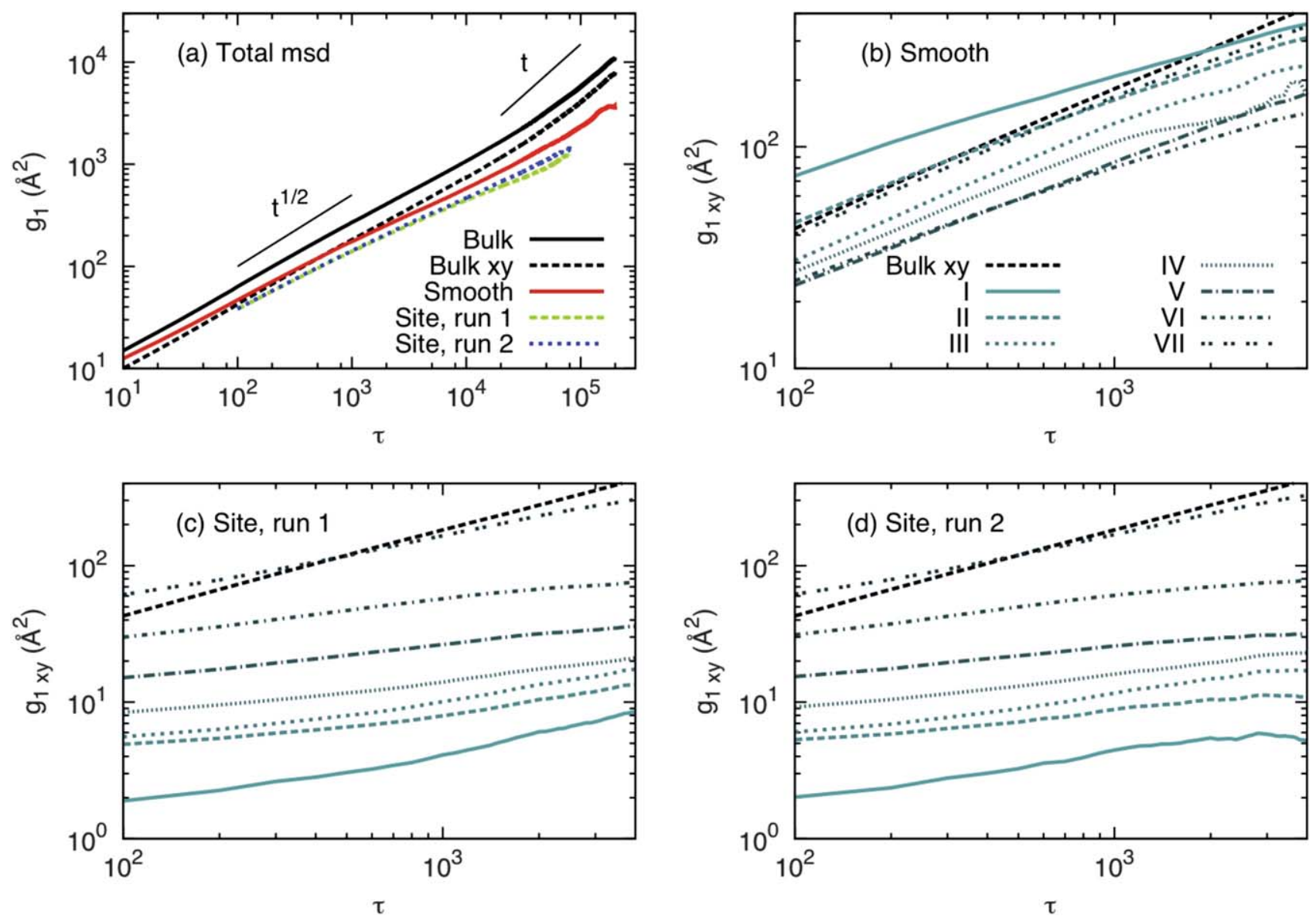

Fig. 10 (a) $g_{1}(\tau)$ for the various walls compared with the bulk. (b)-(d) The local dynamics $g_{1 x y}^{(j)}$ (mean square displacements in $x y$ in region $j$ ) for (b) the smooth wall, (c) run 1 of site-dependent wall, and (d) run 2 of the site-dependent wall. The key for (b) also applies to graphs (c) and (d) and the regions are shown in Fig. 8. In (b)-(d) the mean square displacements in the $x y$ for the bulk system is shown for comparison.

where $j$ is the region, $i$ is the trajectory, $N_{j}$ is the number of segments in region $j$ and $\delta t=t-t_{0}$. Since a new trajectory is started when a particle crosses into another region we can only analyze $g_{x y}^{(i)}$ over a short time scale as the particles change region frequently. All times are also used as an initial time, which improves the statistics significantly for shorter times.

The system was split into seven different regions (or 14 layers) where I is closest to the surface and VII is in the centre, as shown in Fig. 8. The results of this analysis are shown in Fig. 10(b)-(d), where the msd is the average of two corresponding layers $j$ in the system e.g. I is the average of the two surface layers. Although the values in both layers are not identical, the differences between the two layers are much smaller than the differences between the regions. For comparison we have calculated the mean square displacement in the $x y$-plane for the bulk system. For the smooth wall system the dynamics of the surface layer (the P peak) is faster than the bulk dynamics. The increased mobility could be caused by the extra available volume at the interface or local conformation effects, due to the polymer being less entangled. The dynamics of region II is of the same order as the bulk dynamics, regions III-VI show a slowing down of the dynamics compared to the bulk and region VII is again bulk-like. The dynamics of the site-dependent systems are qualitatively different and the surface layer in each site-dependent run is more than an order of magnitude slower than the bulk. As the regions approach the centre of the slab the dynamics of the region approaches the dynamics of the bulk. In the case of the smooth wall potential the msd in layers III and IV becomes noisy after $10^{3} \tau$ because the particles do not remain in this layer for a long time. In layer I, which is closest to the surface, the particles are stuck and remain in the layer for the times considered here. However, the number of particles in this layer is small and the difference in $g_{1 x y}$ for layer I at times $>10^{3} \tau$ between run 1 and run 2 of the site-dependent systems is due to poorer statistics.

\subsection{Diffusion of impurity particles}

Finally the diffusion of impurity particles through the polymer matrix is investigated. Twenty impurity particles the same size as the $P$ beads and with a repulsive surface potential were randomly inserted into the various systems. For each of the surface systems, six independent runs were performed to improve the statistics. The distribution of the impurity particles for the various systems, including bulk, is shown in Fig. 11. The highest concentration of impurities is between the $\mathrm{P}$ and $\mathrm{C}$ peaks at the surface where there is more available volume due to the steric constraints imposed by the surface. Next, there is a depleted region extending approximately $40 \AA$ from the surface. This is 


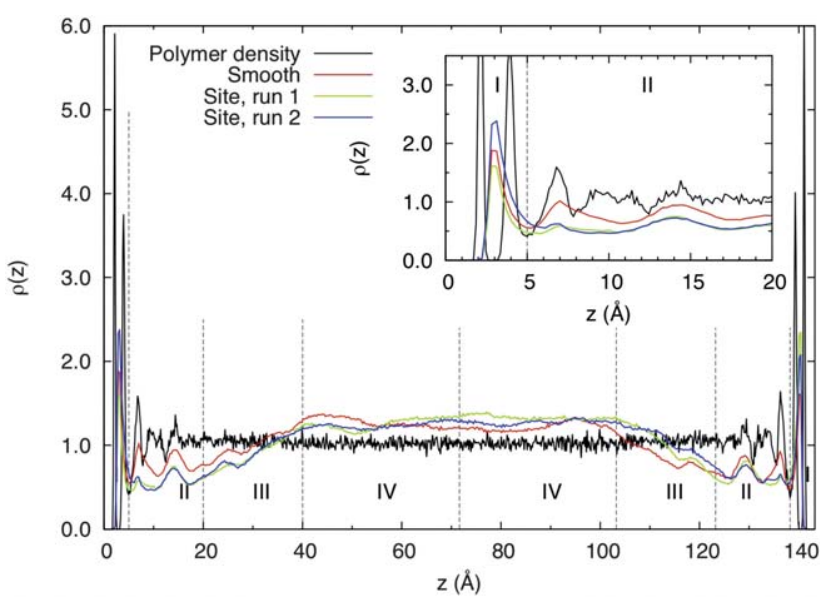

Fig. 11 The normalised concentration profile of impurity particles shown in comparison with the density profile of the polymer for the smooth wall.

approximately the same distance that the density of the chain ends returns to its bulk value. Clearly, the impurity particle is influenced not only by the polymer density but also by the conformations of the chains. This can be seen visually from Fig. 9 where the transition from chains that are adsorbed on the wall to chains that are not adsorbed occurs at around $35-55 \AA$.
We have also calculated $g_{1}$ for the impurity particles in each system. It can be seen from Fig. 12(a) that the average dynamics in each system is not significantly different from the dynamics in the bulk. In the diffusive regime the diffusion coefficient, $D$, is related to $g_{1}$ by the Einstein expression $g_{1}(t)=6 D t$. For diffusion in the bulk we obtain $D=0.50 \AA^{2} \tau^{-1}$. We can estimate the time scaling factor for impurity diffusion between $\mathrm{CG}$ and atomistic simulations by comparing the CG diffusion constant with previous atomistic simulations results of $D \approx 3 \times 10^{-6} \mathrm{~cm}^{2} \mathrm{~s}^{-1}{ }^{23}$ This gives a time scaling factor of $s=16.6 \times 10^{-12} \mathrm{~s}$. Although the scaling factor for impurity particles is a combination of the scaling factors for penetrant and chain diffusion, ${ }^{24}$ the value of $s$ is of the same order of magnitude as the time scaling factor for chain diffusion in bulk BPA-PC, which is $s=30 \times 10^{-12} \mathrm{~s}^{19}$

The $g_{1 x y}^{(i)}$ for the impurity particles can be calculated in the same way as for the polymer diffusion in the previous section. In this case, the slab is divided into four regions, as shown in Fig. 11. The impurity dynamics are not as strongly affected by the surface as the polymer dynamics. Nevertheless, it is clear that the sitedependent surface slows down the dynamics of the impurities in the region next to the surface, and that the value of the dynamics in the centre of the slab approaches the value of the dynamics in bulk. For the smooth wall, the dynamics at the surface are faster than the dynamics in regions II and III, and in region IV the dynamics is similar to that in bulk. Since the region I is small the (a) Total msd

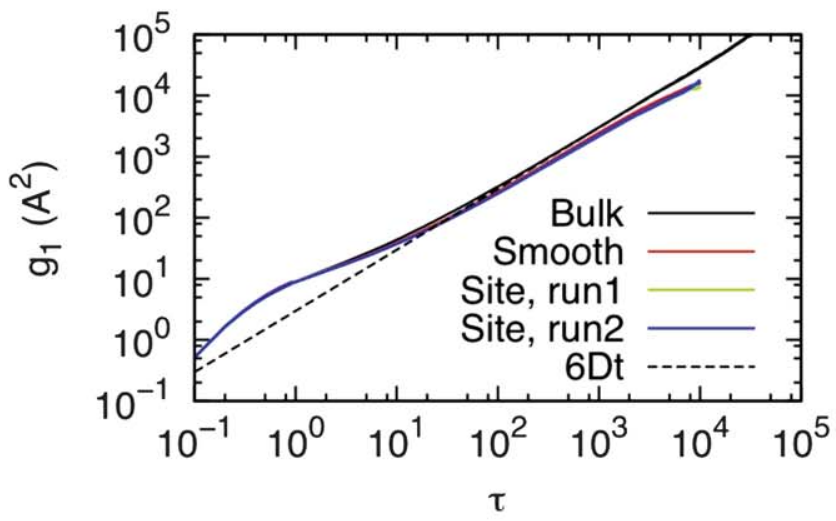

(c) Site, run 1

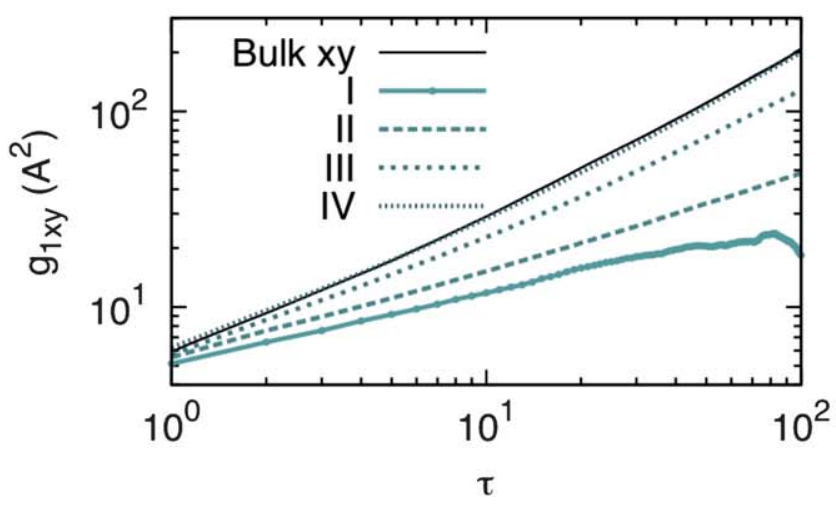

(b) Smooth

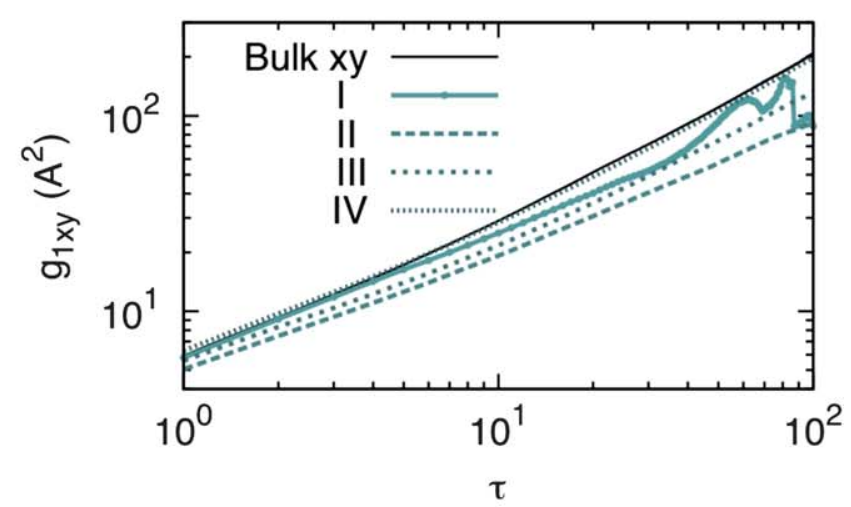

(d) Site, run 2

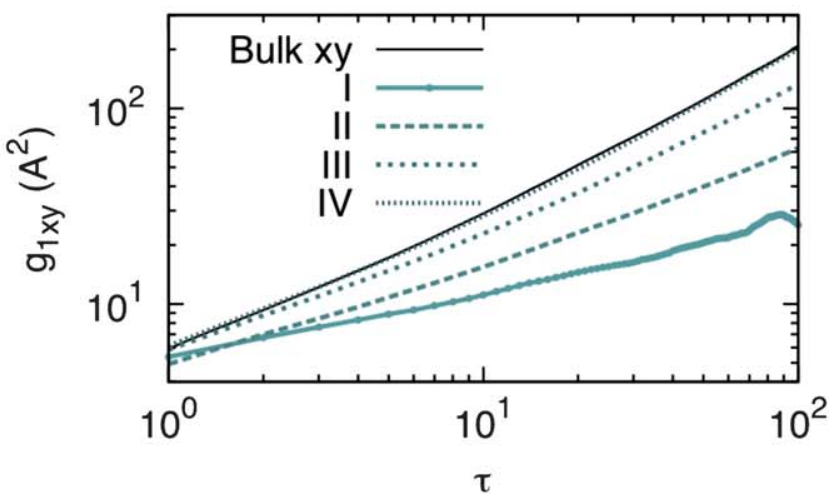

Fig. 12 Mean square displacements for an impurity particle in the regions shown in Fig. 11. (a) $g_{1}(\tau)$ for impurity diffusion in the various wall and bulk systems. (b) $g_{1 x y}^{(j)}$ for the smooth wall system. (c) $g_{1 x y}^{(j)}$ for the site-dependent system, run 1. (d) $g_{1 x y}^{(i)}$ for the site-dependent system, run 2. In (b)-(d) the mean square displacements in the $x y$-plane for the bulk system is shown for comparison. 
time spent in that region is short and the msd is noisy when the time is greater than $\approx 40 \tau$. The local dynamics are shown in Fig. 12(b)-(d).

\section{Discussion and conclusions}

This work presents coarse-grained molecular dynamics simulations of BPA-PC on the silicon $(001)-(2 \times 1)$ surface. The potentials for the surface interactions were based on DFT results of the component beads of the polymer on the surface. Two types of surface potentials were considered: (a) a smooth potential which is dependent only on the distance from the surface, $z$, and (b) a site-dependent potential which is attractive only on sites corresponding to the location of silicon dimers. $z$-dependent angle potentials were added to the $\mathrm{P}$ beads to mimic the adsorption structure of benzene on silicon.

The adsorption structure of BPA-PC on silicon is qualitatively the same as adsorption on nickel with only the chain-end phenylene beads being adsorbed. The structure and density profile is similar for each of the wall types considered and the density reaches the bulk value approximately $20 \AA$ from the surface. From the point of view of the conformations, however, the polymer does not become bulk-like until after $\approx 60 \AA$ from the surface, which is the end-to-end distance for chains with one end attached to the surface. From a dynamical point of view there is a clear difference between the smooth surface and the sitedependent surface. For the smooth surface the dynamics at the surface is slightly faster than the bulk, whereas for the sitedependent surface the mean square displacements are an order of magnitude slower than the bulk.

The diffusion of impurity particles in the various systems was investigated and compared to the diffusion in bulk BPA-PC. The average concentration profile approaches the bulk value at a distance of $\approx 40 \AA$, which corresponds to the transition between chains with both ends attached to the surface and unattached chains as can be seen in Fig. 9. It is, therefore, clear that the impurity particles are sensitive to the conformation of the polymer and not just to the polymer density. As for the polymer dynamics, the site-dependent surfaces slow down the impurity dynamics in the regions next to the surface, whereas for the smooth surface the dynamics of the surface region are faster than in the inner regions. This demonstrates that the use of a smooth surface potential does not adequately describe the dynamics of a polymer next to a strongly site-dependent solid surface, such as silicon.

Finally, we note that there are several ways to improve upon this model. First the surface potentials of the $\mathrm{I}$ and $\mathrm{C}$ beads are approximate for small distances. To improve upon the potentials it would be necessary to consider larger sections of BPA-PC in the DFT calculations in many configurations or even $a b$ initio MD simulations for oligomers. Second, the surface could be more accurately represented by adding structure in the $z$-direction (not just along $x$ and $y$ ) and some directionality could be added to the adsorbed polymer beads. Third, the force constant, $k_{\theta}$, of the $z$-dependent angle potential (the IPC angle) was kept equal to the force constant of this angle in the bulk. If the flexibility of the bond angle in an adsorbed phenylene molecule is higher that for the bulk bond angle this could increase the probability of internal phenylene bead adsorption. However, we believe that this work presents the physically realistic behaviour and that these suggested improvements would result in only minor modifications of the surface interaction.

We would like to thank Luigi Delle Site, Vagelis Harmandaris, Simon Poblete and Nico van der Vegt for useful discussions and Denis Andrienko, Janne Blomqvist, Christine Peter and Torsten Stühn for providing valuable assistance at the early stage of this project. Christine and Denis are also acknowledged for critical reading of the manuscript. Support for Karen Johnston was provided by the Finnish Funding Agency for Technology and Innovation (TEKES), the MMM Initiative of the Max Planck Society and the German Research Foundation (DFG SPP 1369). Additional computational resources were provided by the Center for Scientific Computing (CSC), Finland.

\section{References}

1 L. Delle Site, C. F. Abrams, A. Alavi and K. Kremer, Phys. Rev. Lett., 2002, 89, 156103.

2 L. Delle Site, S. Leon and K. Kremer, J. Am. Chem. Soc., 2004, 126, 2944-2955.

3 L. Delle Site, S. Leon and K. Kremer, J. Phys.: Condens. Matter, 2005, 17, L53-L60.

4 J. Blomqvist and P. Salo, J. Phys.: Condens. Matter, 2009, 21, 225001.

5 K. Johnston and R. M. Nieminen, Phys. Rev. B, 2007, 76, 085402.

6 K. Johnston, J. Kleis, B. I. Lundqvist and R. M. Nieminen, Phys. Rev. $B, 2008,77,121404(\mathrm{R})$.

7 K. Johnston, J. Kleis, B. I. Lundqvist and R. M. Nieminen, Phys. Rev. $B, 2008,77$, (E).

8 L. Delle Site, A. Alavi and C. F. Abrams, Phys. Rev. B, 2003, 67, 193406.

9 H.-J. Limbach, A. Arnold, B. A. Mann and C. Holm, Comput. Phys. Commun., 2006, 174, 704-727.

$10 \mathrm{~S}$. Leon, N. van der Vegt, L. Delle Site and K. Kremer, Macromolecules, 2005, 38, 8078.

11 C. F. Abrams and K. Kremer, Macromolecules, 2003, 36, 260.

12 G. Kresse and J. Furthmüller, Comput. Mater. Sci., 1996, 6, 15.

13 J. P. Perdew, K. Burke and M. Ernzerhof, Phys. Rev. Lett., 1996, 77, 3865.

14 J. Perdew, K. Burke and M. Ernzerhof, Phys. Rev. Lett., 1997, 78, 1396(E).

15 J. P. Perdew, K. Burke, A. Zupan and M. Ernzerhof, J. Chem. Phys., 1998, 108, 1522.

16 P. E. Blöchl, Phys. Rev. B, 1994, 50, 17953.

17 G. Kresse and D. Joubert, Phys. Rev. B, 1999, 59, 1758.

18 K. Johnston, A. Gulans, T. Verho and M. J. Puska, Phys. Rev. B, $2010, \mathbf{8 1}, 235428$

19 B. Hess, S. León, N. van der Vegt and K. Kremer, Soft Matter, 2006, 2, 409.

20 D. Andrienko, S. León, L. Delle Site and K. Kremer, Macromolecules, 2005, 38, 5810-5816.

21 V. A. Harmandaris, K. C. Daoulas and V. G. Mavrantzas, Macromolecules, 2005, 38, 5796.

22 O. Borodin, G. D. Smith, R. Bandyopadhyaya and O. Byutner, Macromolecules, 2003, 36, 7873.

23 O. Hahn, D. A. Mooney, F. Mueller-Plathe and K. Kremer, J. Chem. Phys., 1999, 111, 6061.

24 D. Fritz, C. R. Herbers, K. Kremer and N. F. A. van der Vegt, Soft Matter, 2009, 5, 4556-4563. 